\title{
Chemical Chaperones: A Pharmacological Strategy for Disorders of Protein Folding and Trafficking
}

\author{
DAVID H. PERLMUTTER
}

Departments of Pediatrics, Cell Biology and Physiology, University of Pittsburgh School of Medicine, Children's Hospital of Pittsburgh, Pittsburgh, Pennsylvania

A number of studies published in the last several years have described the use of a group of low-molecular-weight compounds to reverse the mislocalization and/or aggregation of proteins associated with human disease. These compounds that include polyols such as glycerol, trimethylamines such as trimethylamine N-oxide (TMAO) and amino acid derivatives have been called "chemical chaperones." Recent studies have suggested that other compounds, such as 4-phenylbutyric acid (PBA) and membrane-permeable forms of enzyme antagonists, ligands or even substrates, can also act as chemical chaperones for misfolded or mislocalized enzymes. The mechanisms by which chemical chaperones function are not fully understood but are thought to include stabilization of improperly folded proteins, reduction of aggregation, prevention of nonproductive interactions with other resident proteins and alteration of the activity of endogenous chaperones in such a way that the affected proteins are more efficiently transported to the appropriate intracellular or extracellular destination (Fig. 1). Chemical chaperones of the glycerol, TMAO and PBA class have general effects on multiple proteins while antagonists, ligands and substrates are thought to affect only the specific proteins with which they interact.

In addition to having the versatility of general or specific effects, the use of the chemical chaperone strategy is theoretically attractive because of its applicability to a wide range of pathologic conditions. Abnormal localization and/or aggregation of protein has become recognized as a critical issue in a growing number of inherited diseases, including cystic fibrosis,

Received October 4, 2001; Accepted December 20, 2002.

Correspondence to: David Perlmutter, M.D., Department of Pediatrics, Children's Hospital of Pittsburgh, 3705 Fifth Avenue, Pittsburgh, PA 15213-2583; e-mail: perldav@chp.edu

The author's laboratory is supported by the National Institutes of Health.

DOI: 10.1203/01.PDR.0000036878.28942.14 $\alpha 1$-antitrypsin ( $\alpha 1 \mathrm{AT}$ ) deficiency, Alzheimer's disease, prion diseases, familial Parkinsonism, familial forms of amyotrophic lateral sclerosis, spinocerebellar diseases, lysosomal storage diseases as well as in acquired ischemic diseases in which aggregated proteins may contribute to tissue injury (1). In addition to diseases in which abnormal folding may contribute to pathogenesis by a loss of function mechanism (loss of activity, loss of appropriate cellular localization), this strategy is being examined for diseases in which abnormal folding may contribute to pathogenesis by gain-of-function (cytotoxicity of aggregated proteins). Moreover, this strategy has been envisioned as a means for altering physiologic processes for therapeutic purposes, such as improving the efficiency by which antigen is presented by manipulating the assembly of antigenic peptides with MHC molecules. In this review, we will summarize the results of studies in which the chemical chaperone strategy has been tested in cell culture model systems, in experimental animals as well as in patients in vivo.

\section{SMALL ORGANIC COMPOUNDS WITH CHAPERONE-LIKE ACTIVITIES}

Because glycerol and other polyols were known to stabilize proteins against thermal denaturation (2-4) and to facilitate protein folding and oligomeric assembly (5-8), Sato et al. examined the effect of glycerol on the fate of mutant CFTR $\Delta$ F508 (9). Because of its mutation, CFTR $\Delta$ F508, the allele which characterizes $\sim 70 \%$ of all cystic fibrosis patients, is less efficient at reaching the apical plasma membrane of epithelial cells (10). Mutant CFTR molecules are retained in the ER in an immature state and are rapidly degraded by a process that involves the ubiquitin-dependent proteasomal system (11-13). The results of the Sato study showed that $10 \%$ glycerol ( $\mathrm{vol} / \mathrm{vol})$ could mediate an increase in the transport of mutant CFTR molecules from the ER to the plasma membrane 
in a cell culture model system and this effect was associated with an increase in functional activity of CFTR (9). Most importantly, these studies suggested that the effect of glycerol was specific for the folding/maturation of mutant CFTR molecules and not a generalized effect on ER quality control mechanisms. Brown et al. showed that glycerol and two other low molecular weight organic compounds, deuterated water and TMAO, mediated increased posttranslational maturation of CFTR $\triangle F 508$ in a cell culture model system and this was associated with increased chloride transport activity (14). Other studies have shown that chloride transport is increased significantly by the effect of DMSO (DMSO) on polarized epithelial cell lines expressing CFTR $\Delta$ F508 (15). Specific amino acids also appear to affect maturation of CFTR $\Delta$ F508 by a chaperone-like mechanism as evidenced by the effect of glutamine in a cell culture model system of CFTR $\Delta$ F508 (16).

Glycerol, deuterated water, TMAO, DMSO and other low molecular weight compounds, many of which have the property of increasing cellular osmolar activity, partially correct the mislocalization of mutant proteins such as tumor suppressor $\mathrm{p} 53$, viral oncogene $\mathrm{pp} 60^{\mathrm{src}}$, ubiquitin activating enzyme E1 (17), mutant forms of aquaporin-2 associated with nephrogenic diabetes insipidus $(18,19)$, mutant $\alpha 1$-antitrypsin $\mathrm{Z}$ associated with emphysema and liver disease in a $\alpha 1$-antitrypsin deficiency (20) and fragments of the glucocorticoid receptor (21). These data indicate that nuclear, cytoplasmic, plasma membrane and secreted proteins may all be affected. Although many of the affected proteins are temperature-sensitive mutants, several are not (19-21). Other polyols, such as inositol and sorbitol, and combinations of polyols and amino acids, may be even more effective as chemical chaperones for these mutant proteins (WJ Welch, personal communication).

One intriguing recent study has suggested that glycerol, TMAO and DMSO mediate increases in antigen presentation at the cell surface, presumably by enhancing folding and assembly of antigenic peptides with MHC molecules (22). The implication of these observations is that the chemical chaperone strategy could be used to modulate the immune response for therapeutic purposes. These findings also indicate the importance of further studies to determine the margin of safety of these compounds for use in vivo. One could envision the potential for altering physiologic processes, which depend on the assembly and/or biogenesis of multiprotein complexes unselectively. Moreover, one could envision the possibility that at higher concentrations these compounds might alter folding of some molecules in such a way that it actually promoted polymerization/aggregation. In fact, one recent study has shown that glycerol and TMAO accelerate the aggregation amyloid $\beta$-peptide associated with neurodegeneration in Alzheimer's disease (23). In this case chemical chaperones could therefore exacerbate the pathophysiologic state.

The concentrations of glycerol and TMAO needed for chaperone-like effects in cell culture have been in the range of $0.5-1.2 \mathrm{M}$ and $50-100 \mathrm{mM}$, respectively. Studies of glycerol and TMAO given to mice by intraperitoneal, s.c. or oral administration have indicated that it is possible to achieve these concentrations for TMAO but not for glycerol in vivo (24). At a dose of $5 \mathrm{~g} / \mathrm{kg}$ s.c. every $8 \mathrm{~h}$, serum concentrations of TMAO reached $22 \mathrm{mM}$ and urine concentrations of $400 \mathrm{mM}$ (24).

Recent studies by Diamant et al. have suggested the possibility that low molecular weight osmolytes alter the activity of endogenous chaperones (25). These effects are particularly apparent when cells are stressed by high salt concentrations or heat.

\section{4-PHENYLBUTYRIC ACID}

4-Phenylbutyric acid (PBA) is a low-molecular weight fatty acid that has recently been found to have chaperone-like activities. PBA is approved for clinical use as an ammonia scavenger in children with urea cycle disorders (26) and is under evaluation for treatment of thalassemia on the basis of its capacity to activate transcription of $\beta$ - and $\gamma$-globin (27) and for chemotherapy on the basis of its capacity to inhibit histone deacetylase (28). Rubenstein et al. first discovered the chaperone-like activity of PBA when examining its effect on the trafficking of CFTR $\Delta$ F508 to the cell surface (30). They reasoned that PBA, through its transcriptional up-regulating effect, would allow a greater proportion of CFTR $\Delta$ F508 to escape degradation in the ER. A CF bronchial epithelial cell line and primary cultures of nasal polyp epithelium from CF patients were treated with PBA for $7 \mathrm{~d}$ and found to have an increase in CFTR with Golgi-type processing, in cell surface labeling for CFTR and in chloride secretion $(29,30)$. To their surprise, there was no effect on synthesis or degradation. Rubenstein and Zeitlin have since shown that there is a small but significant increase in nasal potential differences reflecting apical surface CFTR function in $18 \mathrm{CF}$ patients treated with PBA for $7 \mathrm{~d}$ at a dose of $19 \mathrm{~g}$ per day (31). Other studies have shown that butyrate may also have chemical chaperone activity for CFTR $\Delta$ F508 (32).

PBA also appears to have chaperone-like activities for the mutant $\alpha 1$-ATZ protein that characterizes $\alpha 1$-AT deficiency. This mutant protein is retained in the endoplasmic reticulum of liver cells rather than secreted into the blood and body fluids in which it ordinarily functions as an inhibitor of neutrophil elastase (33). Lack of $\alpha 1$-AT in the lung allows elastolytic destruction of the connective tissue matrix predisposing deficient individuals to emphysema by a loss-of-function mechanism. Retention of aggregated mutant $\alpha 1$-ATZ in liver cells causes chronic liver injury and a predisposition for hepatocellular carcinoma. Recent studies have shown that PBA mediates an increase in secretion of mutant $\alpha 1$-ATZ in a cell culture model of $\alpha 1$-AT deficiency (20). Oral administration of PBA mediated an increase in blood levels of human $\alpha 1$-AT in a transgenic mouse model of deficiency. Three daily doses were required and well tolerated by the mice. Blood levels of human $\alpha 1$-AT reached $20 \%$ to $50 \%$ of levels present in normal humans. Because the mutant $\alpha 1$-ATZ protein retains a significant degree of elastase inhibitory activity, because clinical studies have suggested that only partial correction is needed for prevention of liver and lung disease in $\alpha 1$-AT deficiency and, because PBA has been used safely in humans, it constitutes a candidate upon which to base chemoprophylactic strategies in $\alpha 1$-AT deficiency. 


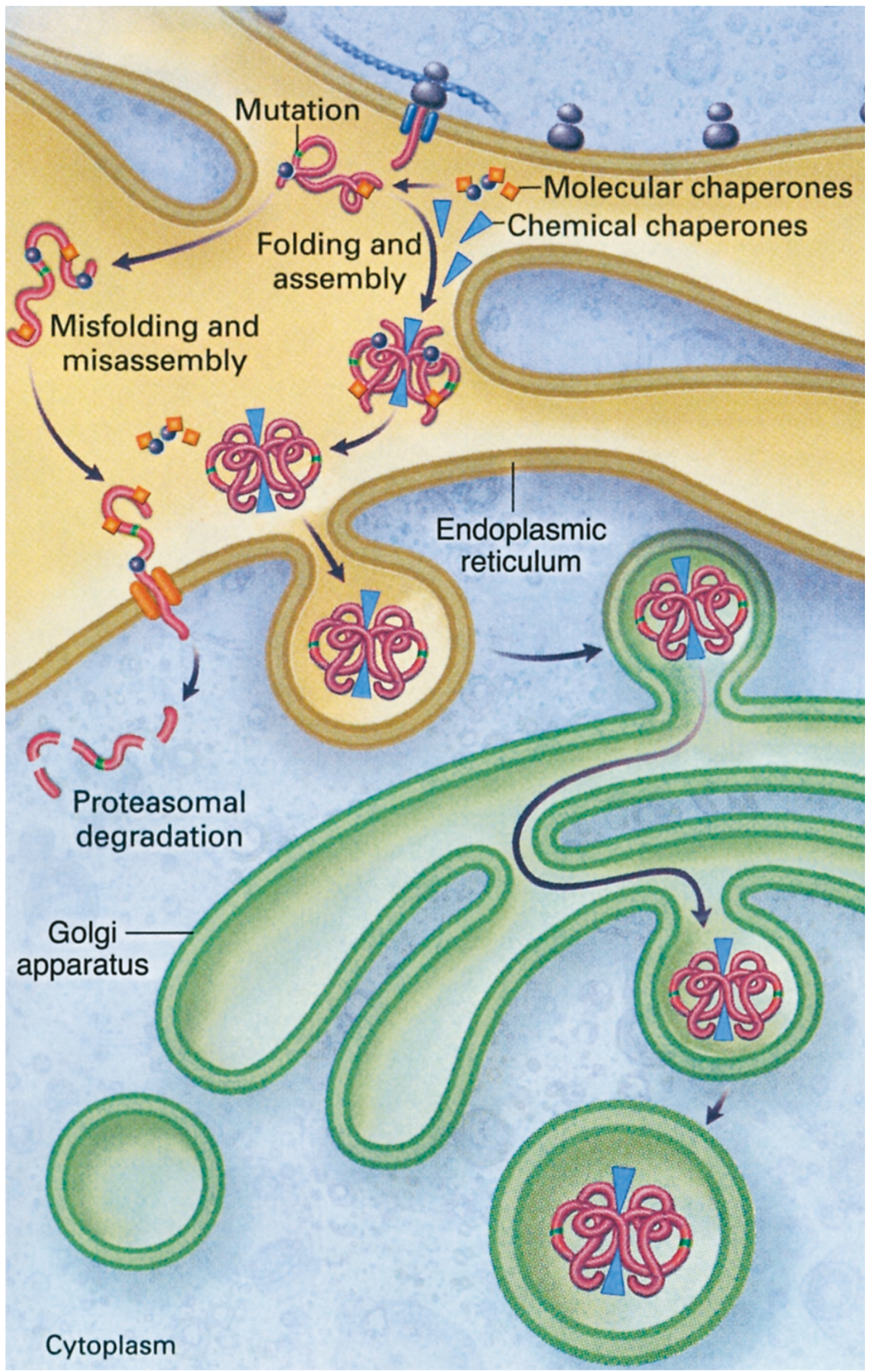




\section{ENZYME ANTAGONISTS}

A new and exciting twist on the concept of chemical chaperone activity has been introduced by Fan et al. (34). These authors examined the effect of a competitive antagonist of $\alpha$-galactosidase A on a mutant form of this lysosomal enzyme that aggregates in the ER of fibroblasts from patients with Fabry disease. Because some competitive antagonists work by occupying the catalytic center of an enzyme and stabilizing its conformation in vitro, Fan et al. reasoned that these antagonists might stabilize the conformation of mutant enzyme in vivo to an extent that would prevent degradation by the quality control apparatus of the ER. The antagonist, 1-deoxygalactonojirimycin (DGJ), when used in subinhibitory concentrations, was found to facilitate the transport of the mutant enzyme from the ER to its usual destination within the lysosome. Cells treated with DGJ have significantly increased galactosidase activity. Oral administration of DGJ to transgenic mice that overexpress mutant $\alpha$ galactosidase A was associated with increased enzyme activity particularly in the heart, spleen and kidney (34). These observations have therefore provided evidence for the concept that when a competitive antagonist works by binding to a functionally active domain of a protein, it may stabilize the conformation of that protein in such a way that translocation through the cell is facilitated. This concept has ramifications for numerous inherited metabolic disorders in which point mutations cause deficiency states as a result of altered conformation.

A subsequent study has shown that antagonists of the vasopressin receptor can mediate increased delivery from the ER to the cell surface of mutant vasopressin receptors associated with nephrogenic diabetes insipidus (35). After the antagonist was removed there was a marked and specific increase in cell surface vasopressin-binding activity. The effect of the antagonists was shown to require intracellular delivery because of a cell-impermeant form of one of the antagonists had no rescue properties. Analysis of the fate of newly synthesized receptors showed that these chemical antagonists facilitated Golgi-type maturation of mutant receptor. Similar results have also been generated for antagonists of potassium channels on mutant channels associated with the long QT syndrome (36).

The paradigm has also been used to enhance assembly and secretion of immunoglobulin using a hapten ligand as chemical chaperone (37). Treatment of a stably transfected cell line with phenylphosphocholine, but not with a nonbinding analog, resulted in a 27-fold increase in secretion of anti-phenylphosphocholine antibody. There was also an increase in antigen binding capacity of the secreted antibody, suggesting that the hapten effect involved stabilization of heavy and light chain assembly.
There are now also several examples of substrates that have chaperone activity for mutant membrane proteins and enzymes. Cell-permeable substrates of P-glycoprotein have been shown to increase cell-surface delivery of a mutant Pglycoprotein as well as increase of its functional activity (38). Frastace $e t$ al. recently administered galactose, a substrate of $\alpha$ galactosidase $\mathrm{A}$, to a patient with the cardiac variant of Fabry disease (39). Previous studies have shown that galactose had a stabilizing effect on mutant forms of galactosidase in cell culture model systems (40). Infusion of galactose once every two days was associated with improvement in cardiac function.

Recent studies by Foster et al. describe several small synthetic compounds that interact specifically with and facilitate folding of temperature-sensitive mutants of the tumor suppressor p53 into active conformation (41). The properties of these compounds indicate that they increase tumor suppressor activity and slow tumor growth in mice. The study also describes strategies for developing high throughput screening assays for libraries of synthetic compounds as a powerful method for developing therapeutic agents that fall into the category of "folding agonist."

It should be noted that antagonists, substrates and the compounds described by Foster et al. involve interactions with specific mutant proteins. This type of chemical chaperone is designed for a specific pathophysiologic situation in each case. The low-molecular-weight organic compounds, polyols, solvents and PBA which provide chemical chaperone activity by general protein stabilizing properties may ultimately be applicable for pathophysiologic states such as ischemia in which there are alterations in folding and/or aggregation of multiple proteins. In either case, the interesting results summarized here provide a compelling argument for further research of these strategies for chemoprophylaxis and/or treatment of several disease states, with the clear-cut need for development of large-scale, high throughput drug screening assays as well as for studies of pharmacokinetics and margin of safety in vivo.

\section{REFERENCES}

1. Kuznesov G, Bush KT, Zhang PL, Nigani SK 1996 Perturbations in maturation of secretory proteins and their association with endoplasmic reticulum chaperones in a cell culture model for epithelial ischemia. Proc Natl Acad Sci USA 93:8584-8589

2. Gekko K, Goa S 1983 Increased thermal stability of collagen in the presence of sugars and polyols. J Biochem 94:199-208

3. Back JF, Oakenfull D, Smith MB 1979 Increased thermal stability of proteins in the presence of sugars and polyols. Biochem 18:5191-5199

4. Gerlsma SY, Stuur ER 1976 The effects of combining urea and an alcohol on the heat-induced reversible denaturation of ribonuclease. Int J Pept Proteins Res 8:3-12

5. Lin PS, Kwock I, Hefter K 1981 Protection of heat induced cytotoxicity by glycerol. J Cell Physiol 108:439-448

6. Henle KJ, Peck JW, Higashikubo R 1983 Protection against heat-induced cell killing by polyols in vitro. Cancer Res 43:1624-1633

7. Denning GM, Anderson MP, Amara JF, Marshall J, Smith AE, Welsh MJ 1992 Processing of mutant cystic fibrosis transmembrane conductance regulator is temperature-sensitive. Nature 358:761-764

\footnotetext{
Figure 1. Putative mechanism of chemical chaperones in the endoplasmic reticulum and Golgi apparatus. Newly synthesized polypeptides (red) are translocated into the lumen of the endoplasmic reticulum. Folding is facilitated by interaction with molecular chaperones (orange, dark blue). If the polypeptide is mutated, there is misfolding and misassembly and then dislocation into the cytoplasm for proteosomal degradation after dissociation of the molecular chaperones. In some cases, but not all, this may involve retrograde translocation through the microsomal import channel. In the presence of chemical chaperones (light blue), folding and assembly of the mutated polypeptide is presumably facilitated so that it can exit the ER by vesicular transport to the Golgi and then from Golgi to plasma membrane and/or extracellular fluid. Molecular chaperones dissociate within the ER but chemical chaperones are associated throughout the secretory pathway because they presumably saturate all of the transport compartments. Modified from Frustaci et al. (39).
} 
8. Restrepo D, Kozody DJ, Spinelli LJ, Knauf PA 1989 Cl-Cl exchange in promyelocytic HL-60 cells follows simultaneous rather than ping-pong kinetics. Am J Physiol 257:C520-C527

9. Sato S, Ward CL, Krouse ME, Wine JJ, Kopito RR 1996 Glycerol reverses the misfolding phenotype of the most common cystic fibrosis mutation. J Biol Chem 271:635-638

10. Cheng SH, Gregory RJ, Marshall J, Paul S, Souza DW, White GA 1990 Defective intracellular transport and processing of CFTR is the molecular basis of most cystic fibrosis. Cell 63:827-834

11. Ward CL, Kopito RR 1994 Intracellular turnover of cystic fibrosis transmembrane conductance regulator. Inefficient processing and rapid degradation of wild-type and mutant proteins. J Biol Chem 269:25710-25718

12. Lukacs GL, Mohamed A, Kartner N, Chang XB, Riordan JR, Grinstein S 1994 Conformational maturation of CFTR but not its mutant counterpart (delta F508) occurs in the endoplasmic reticulum and requires ATP. EMBO J 13:6076-6086

13. Ward CL, Omura S, Kopito RR 1995 Degradation of CFTR by the ubiquitinproteasome pathway. Cell 83:121-127

14. Brown CR, Hong-Brown LQ, Biwersi J, Verkman AS, Welch WJ 1996 Chemical chaperones correct the mutant phenotype of the $\Delta \mathrm{F} 508$ cystic fibrosis transmembrane conductance regulator protein. Cell Stress Chaperones 2:117-125

15. Bebök Z, Venglarik CJ, Panczel Z, Jilling T, Kirk KL, Sorscher EJ 1998 Activation of $\triangle$ F508 CFTR in an epithelial monolayer. Am J Physiol 275:C599-C607

16. Choo-Kang LR, Zeitlin PL 2001 Induction of HSP70 promotes Delta F508 CFTR trafficking Am J Physiol 281:L39-L42

17. Brown CR, Hong-Brown LQ, Welch WJ 1997 Correcting temperature-sensitive protein folding defects. J Clin Invest 99:1432-1444

18. Tamarappoo BK, Verkman AS 1998 Defective aquaporin-2 trafficking in nephrogenic diabetes insipidus and correction by chemical chaperones. J Clin Invest 101:2257-2267

19. Tamarappoo BK, Yang B, Verkman AS 1999 Misfolding of mutant aquaporin-2 water channels in nephrogenic diabetes insipidus. J Biol Chem 274:34825-34831

20. Burrows JAJ, Willis LK, Perlmutter DH 2000 Chemical chaperones mediate increased secretion of mutant $\alpha 1$-antitrypsin $(\alpha 1$-AT) Z: A potential pharmacological strategy for prevention of liver injury and emphysema in $\alpha 1$-AT deficiency. Proc Natl Acad Sci USA 97:1796-1801

21. Baskakov IV, Kumar R, Srinivasan G, Ji WS, Bolen DW, Thompson EB 1999 Trimethylamine $\mathrm{N}$-oxide induced cooperative folding of an intrinsically unfolded transcription-activating fragment of human glucocorticoid receptor. J Biol Chem 274:10693-10696

22. Ghumman B, Bertram EM, Watts TH 1998 Chemical chaperones enhance superantigen and conventional antigen presentation by HLA-DM-deficient as well as HLADM-sufficient antigen-presenting cells and enhance IgG2a production in vivo. J Immunol 161:3262-3270

23. Yang SH, Upis CM, Huang THJ, Chakrabartty A, Fraser PE 1999 Manipulating the amyloid- $\beta$ aggregation pathway with chemical chaperones. J Biol Chem 274:32970-32974

24. Bai C, Biwersi J, Verkman AS, Matthay MA 1999 A mouse model to test the in vivo efficacy of chemical chaperones. J Pharmacol Toxicol Methods 40:39-45
25. Diamant S, Eliahu N, Rosenthal D, Goloubinoff P 2001 Chemical chaperones regulate molecular chaperones in vitro and in cells under combined salt and heat stresses. J Biol Chem 26:39586-39591

26. Maestri NE, Brusilow SW. Clissold DB, Bassett SS 1996 Long-term treatment of girls with ornithine transcarbamylase deficiency. N Engl J Med 335:855-859

27. Collins AF, Pearson HA, Giardina P, McDonagh KT, Crusilow SW, Dover GJ 1995 Oral sodium phenylbutyrate therapy in homozygous beta thalassemia: A clinical trial. Blood 85:43-49

28. Chen WY, Bailey EC, McCune SL, Dong JY, Townes TM 1997 Reactivation of silenced, virally transduced genes by inhibitors of histone deacetylase. Proc Natl Acad Sci USA 94:5798-5803

29. Rubenstein RC, Egan ME, Zeitlin PL 1997 In vitro pharmacologic restoration of CFTR-mediated chloride transport with sodium 4-phenylbutryate in cystic fibrosis epithelial cells containing $\Delta$ F508-CFTR. J Clin Invest 100:2457-2465

30. Rubenstein RC, Zeitlin PL 2000 Sodium 4-phenylbutrate downregulates Hsc70 Implications for intracellular trafficking of $\triangle \mathrm{F} 508-\mathrm{CFTR}$. Am Physiol Soc 278:C259-267

31. Rubenstein RC, Zeitlin PL 1998 A pilot clinical trail of oral sodium 4-phenylbutyrate (Buphenyl) in $\Delta$ F508-homozygous cystic fibrosis patients. Am J Respir Crit Care Med 157:484-490

32. Loffing-Cueni D, Loffing J, Shaw C, Taplin AM, Govindan M, Stanton CR, Stanton BA 2001 Trafficking of GFP-tagged $\triangle$ F508-CFTR to the plasma membrane in a polarized epithelial cell line AM J Physiol Cell Physiol 281:C1889-1897

33. Perlmutter DH 1999 Biology of Disease: Misfolded proteins in the endoplasmic reticulum. Lab Invest 79:623-628

34. Fan JQ, Ishii S, Asano N, Suzuki Y 1999 Accelerated transport and a maturation of lysosomal $\alpha$-galactosidase A in Fabry lymphoblasts by an enzyme inhibitor. Nature Med 5:112-115

35. Morello JP, Salahpour A, Laperrieri A, Bernier V, Arthus MF, Lonergan M 2000 Pharmacological chaperones rescue cell-surface expression and function of misfolded V2 vasopressin receptor mutants. J Clin Invest 105:887-895

36. Zhou Z, Gong Q, January CT 1999 Correction of defective protein trafficking of a mutant HERG potassium channel in human long QT syndrome. J Biol Chem 274:31123-31126

37. Wiens GD, O'Hare T, Rittenberg MB 2001 Recovering antibody secretion using a hapten ligand as a chemical chaperone. J Biol Chem 276:40933-40939

38. Loo TW, Clarke DM 1997 Correction of defective protein kinesis of human Pglycoprotein mutants by substrates and modulators. J Biol Chem 272:709-712

39. Frustaci A, Chimenti C, Ricci R, Natale L, Russo MA, Pieroni M 2001 Improvement in cardiac function in the cardiac variant of Fabry's disease with galactose-infusion therapy. N Engl J Med 345:25-32

40. Okumiya T, Ishii S, Takenda T 1995 Galactose stabilizes various missense mutants of $\alpha$-galactosidase in Fabry disease. Biochem Biophys Res Commun 214:1219-1224

41. Foster BA, Coffey HA, Morin MJ, Rastinejad F 1999 Pharmacological rescue of mutant p53 conformation and function. Science 286:2507-2510 